\title{
Prevalence of statin utilization and adherence among privately insured subjects in the Commonwealth of Puerto Rico
}

\author{
José J Hernández-Muñoz, PhD; Emily S Wong, PharmD Candidate; \\ and Chandni R Kamdar, PharmD Candidate
}

\section{What is already known about this subject}

- Puerto Ricans living on the U.S. mainland have the highest age-, sex-, and body mass index-adjusted prevalence of diagnosed diabetes among Hispanics (16.7\%) and are the highest at-risk group for developing cardiovascular disease.

- Puerto Ricans are the Hispanic subgroup with the highest age- and sex-adjusted prevalence of statineligible patients (20.9\%).

- Only $36.0 \%$ of insured Puerto Ricans at high risk for cardiovascular disease in the United States are using statins.

\section{ABSTRACT}

BACKGROUND: Puerto Ricans are the Hispanic subgroup with the highest adjusted prevalence of statin-eligible patients. However, no study has described statin utilization and adherence among subjects living on the island of Puerto Rico.

OBJECTIVES: To (a) estimate the prevalence of beneficiaries with diabetes aged between 40 and 75 years; (b) estimate the prevalence of statin utilization among beneficiaries with diabetes; and (c) estimate secondary adherence to statins among beneficiaries with diabetes.

METHODS: With pharmacy claims data from a commercial pharmacy benefit manager (PBM) in the Commonwealth of Puerto Rico, this study used a retrospective longitudinal

\section{What this study adds}

- Among a group of privately insured patients aged between 40 and 75 years in the Commonwealth of Puerto Rico, the prevalence of diabetes is $7.8 \%$.

- Among the same group, the proportion of patients with diabetes who are using statins is $57.1 \%$.

- The median proportion of days covered (PDC) for statins among this group was $63.4 \%$, and only $27.5 \%$ achieved a PDC $\geq 80 \%$.

\section{Author affiliations \\ Jose J Hernández-Muñoz, PhD; Emily S Wong, PharmD Candidate; Chandni R Kamdar, PharmD Candidate, Irma L Rangel College of Pharmacy, Texas A\&M University, College Station, TX.}

\section{AUTHOR CORRESPONDENCE:} Chandni R Kamdar, 832.704.0206; kamdar@tamu.edu

J Manag Care Spec Pharm. 2021;27(3):392-98

Copyright $(2021$, Academy of Managed Care Pharmacy. All rights reserved.

design to analyze all pharmacy claims generated by 115,674 beneficiaries aged between 40 and 75 years with continuous enrollment during 2018. Beneficiaries with diabetes were defined by having $\geq 2$ pharmacy claims for antidiabetic agents during 2018. Statin utilization was defined by having $\geq 1$ pharmacy claim for statins among beneficiaries with diabetes. The proportion of days covered (PDC) was used to measure secondary adherence to statins. Parametric and nonparametric statistics were used to describe statin utilization and adherence.

RESULTS: The prevalence of beneficiaries with diabetes was $7.8 \%$. Of the 8,975 beneficiaries with diabetes, 5,129 (57.1\%) received $\geq 1$ prescription for a statin. Older males with diabetes were more likely to receive prescriptions for statins. The median PDC for the
4,553 beneficiaries with $\geq 2$ prescriptions for statins was $63.4 \% ; 3,306$ (72.6\%) beneficiaries filled their statin prescriptions for a 30-day supply only; and 1,252 (27.5\%) beneficiaries had a PDC $\geq 80 \%$. The highest PDC (92.3\%) was observed for beneficiaries who received statins for a 90-day supply only.

CONCLUSIONS: This is the first study that has measured statin utilization and adherence among patients with diabetes living in Puerto Rico. The utilization and adherence to statins among privately insured beneficiaries with diabetes in Puerto Rico are suboptimal. Future studies should focus on understanding the reasons for the suboptimal use of statins and on potential interventions at the beneficiary and provider level to increase statin utilization. 
The 2018 American College of Cardiology/American Heart Association Guideline on the Management of Blood Cholesterol and the 2018 American Diabetes Association Cardiovascular Disease and Risk Management: Standards of Medical Care in Diabetes recommend statins for primary prevention to reduce atherosclerotic cardiovascular disease (ASCVD) in patients with diabetes who are aged between 40 and 75 years. ${ }^{1,2}$ Adherence to statins has been associated with fewer cardiovascular hospitalizations, fewer emergency department visits, and decreased medical costs. ${ }^{3-5}$ Unfortunately, nationally representative studies from the United States have shown a suboptimal prevalence of statin use and adherence among patients with diabetes who qualify for a statin medication, ${ }^{6-11}$ especially among subgroups with a lower perceived risk of cardiovascular events, such as patients taking statins for primary prevention. ${ }^{8,9}$

Hispanics have consistently been shown to have one of the highest prevalence of diabetes among all ethnic groups in the United States. ${ }^{12}$ Therefore, they should maximize the benefits associated with statin utilization. However, several studies have highlighted the underutilization and suboptimal adherence to statins among Hispanics, as compared with other ethnic groups..$^{6-8,10,13,14} \mathrm{~A}$ significant limitation of these studies is the lack of differentiation between statin utilization and adherence by Hispanic subgroups. ${ }^{14-17}$ Sociodemographic and socioeconomic heterogeneity between and within Hispanic subgroups makes extrapolating findings from Hispanics to their corresponding subgroups and from subgroups to their corresponding elements potentially inaccurate. ${ }^{18-20}$ A study of individuals at high risk for cardiovascular disease living in the United States reported a suboptimal prevalence of statin utilization for all Hispanic subgroups, ranging from 26.5\% for insured individuals from South America to $36.0 \%$ for insured individuals from Puerto Rico. ${ }^{21}$ Puerto Ricans living in the United States also have been identified as the Hispanic subgroup with the highest age- and sex-adjusted prevalence of statin eligibility. ${ }^{22}$

Among Hispanics in the United States, Puerto Ricans have the highest risk for cardiovascular disease (26\%) and the highest age-, sex-, and body mass index-adjusted prevalence of diagnosed diabetes (16.7\%). ${ }^{21,23}$ Although a few large-scale studies have documented the underutilization of statins among Puerto Ricans living on the U.S. mainland, ${ }^{21,22}$ no study has documented statin utilization and adherence for those living on the island of Puerto Rico.

The objectives for this study were to (a) estimate the prevalence of beneficiaries with diabetes among enrollees of a pharmacy benefit management company (PBM) in
Puerto Rico aged between 40 and 75 years; (b) estimate the prevalence of statin utilization among beneficiaries with diabetes; and (c) determine the adherence levels to statin therapy using the proportion of days covered (PDC).

\section{Methods}

\section{STUDY DESIGN AND SAMPLE}

This study used a 1-year retrospective longitudinal design with claims data from a commercial PBM in Puerto Rico. Privately insured beneficiaries with continuous enrollment, aged between 40 and 75 years, and $\geq 2$ pharmacy claims for an antidiabetic therapy during 2018 were included as the study sample. The PBM manages coverage for approximately 250,000 beneficiaries from all geographical regions on the island annually. The study was classified as exempt by the institutional review board. A list of the Generic Product Identifier codes used to capture pharmacy claims for antidiabetic agents and statins is included in Table 1.

\section{STUDY VARIABLES}

Several variables were extracted from the pharmacy claims dataset for all of the beneficiaries in the study sample. Sociodemographic variables included age and gender. Age was defined as a beneficiary's age by the date of the first prescription for an antidiabetic agent during the study period. Other variables included the number and days supply of prescription claims for antidiabetic agents and statins. Data were collected for the number of physicians who prescribed statins per beneficiary and the number of pharmacies that filled prescriptions for statins per beneficiary during the study period.

\section{PREVALENCE OF DIABETES}

The prevalence of diabetes was calculated by dividing the number of beneficiaries with $\geq 2$ pharmacy claims for an antidiabetic therapy by the total number of beneficiaries in the PBM aged between 40 and 75 years in 2018.

\section{STATIN UTILIZATION}

Prevalence of statin utilization was calculated by dividing the number of beneficiaries with $\geq 1$ pharmacy claim for a statin by the number of beneficiaries with diabetes aged between 40 and 75 years during 2018. The statin prescriptions may have occurred at any time during the study period.

\section{STATIN ADHERENCE}

Secondary adherence to statins was calculated using PDC and the PDC threshold. PDC is a well-established method 


\section{TABLE 1 Generic Product Identifier Codes to Capture Claims for Antidiabetic Agents and Statins}

\begin{tabular}{l|c}
\hline \hline Drug Class: Antidiabetics & GPI 4 \\
\hline Incretin mimetic agents $^{\text {a }}$ & 2717 \\
\hline Sulfonylureas & 2720 \\
\hline Biguanides & 2725 \\
\hline Meglitinide analogues & 2728 \\
\hline Alpha-glucosidase inhibitors & 2750 \\
\hline Dipeptidyl peptidase 4 inhibitors & 2755 \\
\hline Dopamine receptor agonists & 2757 \\
\hline Insulin sensitizing agents & 2760 \\
\hline Sodium glucose co-transporter 2 inhibitors & 2770 \\
\hline Antidiabetic combinations & 2799 \\
\hline Insulins & 2710 \\
\hline Amylin analogues & 2715 \\
\hline Drug Class: Statins & GPI 4 \\
\hline HMG CoA reductase inhibitors ${ }^{b}$ & 3940 \\
\hline Drug Class: Statin Combination Products & GPI 6 \\
\hline Ezetimibe and simvastatin & 399940 \\
\hline Atorvastatin and amlodipine & 409925 \\
\hline${ }^{a}$ GLP-1 receptor agonists. & \\
\hline HMG-CoA $\beta$-Hydroxy $\beta$-methylglutaryl-CoA. & \\
\hline
\end{tabular}

for measuring adherence using claims data. ${ }^{24}$ Beneficiaries included in the analysis were patients with diabetes who had $\geq 2$ pharmacy claims for a statin. The denominator for PDC considered the number of days that a beneficiary was eligible to receive statin prescriptions during the study period. A beneficiary was eligible to receive a prescription for a statin after the first fill of $\geq 2$ prescriptions until December 31, 2018. The numerator included the actual number of days covered with a statin prescription after the first fill of $\geq 2$ prescriptions statin medication until December 31, 2018. A PDC threshold of $\geq 80 \%$ was categorized as adherent, and $<80 \%$ as nonadherent, to statin therapy. PDC thresholds $>100 \%$, due to the carryover effect of the days supply, were truncated to $100 \%$.

\section{STATISTICAL ANALYSIS}

All beneficiaries who complied with the diabetes definition for this study were described by age, gender, number of paid prescriptions for antidiabetic agents, and whether they filled $\geq 1$ prescription for a statin during the study period. Statistical comparisons for the distribution of the variables previously listed were made for beneficiaries with diabetes who filled $\geq 1$ prescription for statins and those who did not. Beneficiaries who filled $\geq 1$ prescription for statins were described by the number of days supply (i.e., 15, 30, 30 and $90,90$, or $<30$, or other than 15$)$; the number of pharmacies used to fill their statin prescriptions (i.e., 1,2 , or $\geq 3$ pharmacies); and the number of medical providers who prescribed their statins (i.e., 1,2 , or $\geq 3$ providers).

The median PDC and corresponding interquartile range (IQR) were calculated for beneficiaries with $\geq 2$ prescriptions for statins. Beneficiaries were classified by their PDC thresholds (i.e., $\geq 80 \%$ or $<80 \%$ ) and compared for each of the study variables previously listed. To identify statistically significant differences in the bivariate analyses, Student t-tests were used to compare the means for variables that followed a normal distribution, and the Mann-Whitney $U$ and Kruskal-Wallis tests were used to compare the medians for variables with a non-normal distribution. The Dwass, Steel, Critchlow-Fligner method was used for the pairwise 2-sided multiple comparisons, and the chi-square test was used when both variables were measured categorically.

\section{Results}

\section{PREVALENCE OF DIABETES}

A total of 115,674 beneficiaries aged between 40 and 75 years were enrolled in the PBM during 2018. Of the 115,674 beneficiaries, 8,975 had $\geq 2$ pharmacy claims for antidiabetic agents, with a prevalence of diabetes of $7.8 \%$. For the 8,975 beneficiaries with diabetes, the mean (SD) age was 55 (8) years, and 4,781 (53.3\%) were males. The median (IQR) for the number of fills for antidiabetic agents was 9 (5-13).

\section{STATIN UTILIZATION}

Of the 8,975 beneficiaries with diabetes, 5,129 (57.1\%) filled $\geq 1$ prescription for a statin. Their mean (SD) age was 56 (7) years and, on average, 3 years older than those who did not fill a prescription for a statin $(\mathrm{P}<0.0001)$. A total of 2,773 (54.1\%) beneficiaries were males, and although diabetic males had higher odds of using statins than females, the difference was not statistically significant (odds ratio $[\mathrm{OR}]=1.07,95 \% \mathrm{CI}=0.99-1.17)$. Among the 5,129 beneficiaries who filled $\geq 1$ statin prescription, the median (IQR) number of prescriptions for statins was 5 (3-9). A total of 3,635 (70.9\%) beneficiaries filled their statin prescriptions for a 30 -day supply only, and 717 (14.0\%) filled for a 90-day supply only. On the other hand, 269 (5.2\%) beneficiaries filled their statin prescriptions for 30- and 90-days supply; 465 (9.1\%) filled for a 15-day supply only; and 43 (0.8\%) filled for less than 


\section{TABLE 2 The Median PDC Distribution $(\mathrm{N}=4,553)$}

\begin{tabular}{|c|c|c|c|c|}
\hline Variable & $\mathbf{N}(\%)$ & $\begin{array}{c}\text { Median PDC } \\
\%(\text { IQR) }\end{array}$ & $\begin{array}{l}\text { Overall } \\
\text { P Value }\end{array}$ & $\begin{array}{c}\text { Multiple Comparisons } \\
\text { P Value }\end{array}$ \\
\hline \multicolumn{5}{|l|}{ Gender } \\
\hline Males & $2,485 \quad(54.6)$ & $65.7(44.9-83.3)$ & \multirow[t]{2}{*}{$0.0012^{\mathrm{b}}$} & \multirow[t]{2}{*}{-} \\
\hline Females & $2,068 \quad(45.4)$ & $61.7(42.3-80.8)$ & & \\
\hline
\end{tabular}

Days supply for statin prescriptions, $n=4,511^{\mathrm{c}}$

\begin{tabular}{l|rr|l|l|rr}
\hline 15 days only & 464 & $(10.3)$ & $26.5(15.3-40.8)$ & $<0.0001^{d}$ & $(15$ vs. 30$)<0.0001$ \\
\hline 30 days only & 3,306 & $(73.3)$ & $65.9(47.6-80.8)$ & & $(15$ vs. $30 / 90)<0.0001$ \\
\hline 30 or 90 days & 269 & $(5.9)$ & $58.3(42.1-77.4)$ & & $(15$ vs. 90$)<0.0001$ \\
\hline 90 days only & 472 & $(10.5)$ & $92.3(74.9-100.0)$ & & $(30$ vs. $30 / 90)$ & 0.0953 \\
\hline & & & & & $(30$ vs. 90$)<0.0001$ \\
\hline & & & & & $(30 / 90$ vs. 90$)<0.0001$ \\
\hline
\end{tabular}

Number of pharmacies visited to fill statins

\begin{tabular}{l|rr|r|r|rr}
\hline 1 & 3,725 & $(81.8)$ & $62.9(42.5-82.2)$ & $0.0628^{d}$ & $(1$ vs. 2$)$ & 0.1865 \\
\hline 2 & 728 & $(16.0)$ & $65.8(47.5-82.6)$ & & $(1$ vs. $\geq 3)$ & 0.2143 \\
\hline$\geq 3$ & 100 & $(2.2)$ & $67.6(53.8-79.4)$ & & $(2$ vs. $\geq 3)$ & 0.5832 \\
\hline
\end{tabular}

Number of medical providers who prescribed statins

\begin{tabular}{l|rc|c|r|rr}
\hline 1 & 2,886 & $(63.3)$ & $62.5(43.2-81.1)$ & $0.1101^{\text {d }}$ & (1 vs. 2) & 0.1176 \\
\hline 2 & 1,291 & $(28.4)$ & $65.2(45.0-83.1)$ & & $(1$ vs. $\geq 3)$ & 0.5312 \\
\hline$\geq 3$ & 376 & $(8.3)$ & $65.0(42.9-85.7)$ & & $(2$ vs. $\geq 3)$ & 0.9986 \\
\hline
\end{tabular}

a Dwass, Steel, Critchlow-Fligner method for pairwise 2-sided multiple comparisons.

${ }^{b}$ Mann-Whitney $U$ test.

${ }^{c} n=42$ beneficiaries with statin prescriptions for $<30$-day supply, other than those with a 15-day supply, were excluded.

${ }^{d}$ Kruskal-Wallis tests.

$I Q R=$ interquartile range; $P D C=$ proportion of days covered .

a 30-day supply, excluding those with a 15-day supply. Most beneficiaries, 4,301 (83.9\%), visited only 1 pharmacy to fill all their statin prescriptions; 728 (14.2\%) visited 2 pharmacies; and $100(1.9 \%)$ visited 3 or more. Similarly, 3,854 (75.1\%) beneficiaries received all of their statins prescriptions from the same medical provider; 1,080 (21.1\%) received them from 2 different providers; and 195 (3.8\%) received their prescriptions from 3 or more providers.

\section{STATIN ADHERENCE}

Of the 5,129 beneficiaries with $\geq 1$ prescription for a statin, 4,553 (88.8\%) had $\geq 2$ prescriptions for a statin and were included in the PDC calculation. The median (IQR) PDC was 63.4\% (44.6$82.2)$, and $1,252(27.5 \%)$ beneficiaries had a $\mathrm{PDC} \geq 80 \%$. Their mean (SD) age was 56 (7) years. However, age was not a factor that predicted a $\mathrm{PDC} \geq 80 \%$ $(P=0.734)$. Males had higher odds of achieving a $\mathrm{PDC} \geq 80 \%$ than females (OR=1.17, 95\% CI=1.10-1.34). The 4,553 beneficiaries included in the PDC analysis filled their statins at the following proportions: 464 (10.2\%) 15-day supply only, 3,306 (72.6\%) 30-day supply only, 269 (5.9\%) 30- and 90-day supply, 472 (10.4\%) 90-day supply only, and $42(0.9 \%)<30$-day supply, excluding those with a 15-day supply. The median (IQR) PDC for beneficiaries who filled their prescriptions for a 15 -day supply only was $26.5 \%$ (15.340.8 ); a 30 -day supply only was $65.9 \%$ (47.6-80.8); a combination of a 30-day and 90-day supply was 58.3\% (42.177.4 ); and a 90-day supply only was 92.3\% (74.9-100.0). The overall median PDC variation by days supply was statistically significant $(P<0.0001)$.

The pairwise 2-sided multiple comparisons for the median PDC between the 4 groups led to statistically significant results $(P<0.0001)$, with the exclusion of the PDC comparison between the 30-day supply only (65.9\%) and the 30-day and 90-day supply groups $(58.3 \% ; \mathrm{P}=0.0953)$. The median (IQR) PDC for the beneficiaries who filled their statin prescriptions from a single pharmacy was $62.9 \%$ (42.5-82.2), from 2 different pharmacies $65.8 \%$ (47.5-82.6), and 3 or more pharmacies 67.6\% (53.8-79.4). However, the differences between the 3 median PDC $(P=0.0628)$ and the pairwise 2-sided multiple comparisons between the 3 groups were not statistically significant (all $P \geq 0.1000$ ). Also, the beneficiaries who received their statin prescriptions from a single provider had a median (IQR) PDC of $62.5 \%$ (43.2-81.1); those from 2 different providers was $65.2 \%$ (45.0-83.1); and those from 3 or more providers was $65.0 \%$ (42.9-85.7). The difference between the 3 median PDC $(P=0.1101)$ and the pairwise 2-sided multiple comparisons between the 3 groups were not statistically significant (all $P \geq 0.1000$; Table 2).

\section{Discussion}

The prevalence of beneficiaries with diabetes was $7.8 \%$ when using the criterion of $\geq 2$ antidiabetic claims. The decision to use $\geq 2$ claims added robustness to the identification process without introducing significant 
deviations in the estimated prevalence. The estimated prevalence was lower than the age-adjusted prevalence reported for island Puerto Ricans in 2016 (13.7\%). ${ }^{12}$ It should be highlighted that in the current study, the prevalence of diabetes was based on a group of privately insured patients. Therefore, groups that may have a higher prevalence of diabetes, such as those within the lowest poverty income ratio, were not included. ${ }^{25} \mathrm{Also}$, patients with diabetes who did not receive pharmacological treatment from their physicians or who decided not to fill their diabetes medication may have caused an underestimation of the true prevalence. Case ascertainment (i.e., self-reported vs. pharmacy claims) could also explain the differences in the prevalence of diabetes.

Despite treatment guideline recommendations to treat diabetes patients aged between 40 and 75 years with statins as the primary prevention to reduce ASCVD in the current study, ${ }^{1,2}$ only $57.1 \%$ of the diabetic patients received $\geq 1$ prescription for a statin. This is the first study that measured the prevalence of statin utilization among diabetic patients in Puerto Rico; therefore, there is no benchmark for comparison. However, a study among Hispanics at high risk for cardiovascular disease on the U.S. mainland reported a prevalence of statin utilization of $36.0 \%$ among insured Puerto Ricans aged between 18 and 74 years. ${ }^{21}$ Nationally representative studies in the United States have reported an overall prevalence of statin use among patients with diabetes aged between 40 and 75 years ranging from $38 \%$ to $59 \%$ and $29 \%$ to $45 \%$ among Hispanics. ${ }^{6-8,10}$ In our study the prevalence of statin use is closer to the overall U.S. prevalence and higher than the prevalence among Hispanics and Puerto Ricans on the U.S. mainland. Similar to previous studies, the current study found that male and older patients with diabetes had a higher chance of receiving $\geq 1$ statin prescription. . $^{910}$

Our study reported a median PDC for statins of $63.4 \%$. The estimated PDC is slightly higher than that reported for patients with diabetes from U.S. commercial databases (56\%-60\%). ${ }^{14,26}$ The lack of studies reporting PDC for statins among Hispanics with diabetes limits the comparison of our findings to similar ethnic groups and subgroups. Despite displaying a better PDC, the current study found that only $27.5 \%$ of the beneficiaries had a PDC $\geq 80 \%$. This number is lower than that reported for patients with diabetes from U.S. commercial databases from 2007 to 2014 (35\%-38\%). ${ }^{14}$

Although visiting multiple pharmacies has been associated with lower PDC values for statins, ${ }^{27}$ our study suggests that using $>1$ pharmacy to fill the statin prescriptions or receiving the statin prescriptions from $>1$ physician had no statistically significant effect on PDC values. Beneficiaries who filled their statin prescriptions for a 90-day supply had the highest PDC. Although clinically this does not translate into beneficiaries taking their statins every day for 90 days, at minimum the patient has direct access to take it as directed. While the PBM allowed for 90-day fills at a zero copay for low-intensity statins, our study corroborated previous findings suggesting that Hispanics prefer to fill their antihyperlipidemic therapies for $\leq 30$-day fills. ${ }^{28}$

\section{LIMITATIONS}

Our study has several limitations. First, filling a prescription is not synonymous with use. Second, we were not able to differentiate if nonuse was because of physician or beneficiary beliefs. Previous studies have reported varying beliefs among primary care providers about the risks and benefits of statin therapy for primary prevention. ${ }^{29}$ Other studies have reported that $15 \%$ of patients who receive prescriptions for a statin do not pick up the prescription from the pharmacy within 90 days of the order date..$^{30}$ Therefore, interventions to improve statin use should focus on the patient's determinant of health and the prescriber's role in the process.

Third, we focused on privately insured patients, so our findings are not representative of the approximately 1.5 million lives under the Mi Salud Healthcare Plan, a federally funded health insurance program in Puerto Rico. ${ }^{31}$ Fourth, we did not have data on statin strength in order to determine statin intensity, so we were not able to establish appropriateness of statin prescribing.

Finally, the denominator for the statin PDC calculation varied by the beneficiary. However, when conducting a sensitivity analysis including only beneficiaries with $\geq 100$ days, $\geq 200$ days, or $\geq 300$ days of statin eligibility during the study period, the corresponding median PDCs were $62.6 \%, 62.1 \%$, and $63.8 \%$, respectively. Therefore, variations in the statin eligibility periods did not affect the estimated median PDC for statins.

\section{Conclusions}

The utilization and adherence to statins in a group of privately insured subjects with diabetes in Puerto Rico are suboptimal. However, they are higher than that reported for insured Puerto Ricans with diabetes in the United States. Caution should be exercised when trying to label Hispanics as a homogenous ethnic group or to extrapolate findings from Puerto Ricans on the U.S. mainland when compared with those on the island. Future studies should 
focus on understanding the reasons for the suboptimal use of statins and on potential interventions at the beneficiary and provider level to increase statin utilization.

\section{DISCLOSURES}

No outside funding supported this study. The authors have no conflicts of interest or financial disclosures to disclose related to this study.

\section{ACKNOWLEDGMENTS}

The authors thank Ashley Parambil, Toney Duong, Ishimwe Masozera, and Uyen Hoang, pharmacy students from the Texas A\&M University Irma L. Rangel College of Pharmacy, for their contributions during the preparation phase of the manuscript. The authors also thank Dr. Natalie Rosario, Clinical Assistant Professor at the Texas A\&M University Irma L. Rangel College of Pharmacy, for her clinical review and comments on the final draft of the manuscript. Thanks are also extended to Dr. Martty Martínez-Fraticelli, Dr. Iris V. RománBermúdez, and Mrs. Leidy Betancourt for their clinical and technical support regarding the pharmacy claims data used to conduct this study.

\section{REFERENCES}

1. Grundy SM, Stone NJ, Bailey AL, et al. 2018 AHA/ACC/AACVPR/AAPA/ABC/ ACPM/ADA/AGS/APhA/ASPC/NLA/ PCNA Guideline on the Management of Blood Cholesterol: a report of the American College of Cardiology/American Heart Association Task Force on Clinical Practice Guidelines. J Am Coll Cardiol. 2019;73(24):e285-e350.

2. American Diabetes Association. 9. Cardiovascular disease and risk management: Standards of Medical Care in Diabetes - 2018. Diabetes Care. 2018;41(Suppl 1):S86-S104.

3. Franklin JM, Krumme AA, Tong AY, et al. Association between trajectories of statin adherence and subsequent cardiovascular events. Pharmacoepidemiol Drug Saf. 2015;24(10):1105-13.
4. Wu J, Seiber E, Lacombe VA, Nahata MC, Balkrishnan R. Medical utilization and costs associated with statin adherence in Medicaid enrollees with type 2 diabetes. Ann Pharmacother. 2011;45(3):342-49.

5. Zhao Y, Zabriski S, Bertram C. Associations between statin adherence level, health care costs, and utilization. J Manag Care Spec Pharm. 2014;20(7): 703-13. doi: 10.18553/jmcp.2014.20.7.703

6. Adedinsewo D, Taka N, Agasthi P, Sachdeva R, Rust G, Onwuanyi A. Prevalence and factors associated with statin use among a nationally representative sample of U.S. adults: National Health and Nutrition Examination Survey, 2011-2012. Clin Cardiol. 2016;39(9):491-96.

7. Wong ND, Young D, Zhao Y, et al. Prevalence of the American College of Cardiology/American Heart Association statin eligibility groups, statin use, and low-density lipoprotein cholesterol control in U.S. adults using the National Health and Nutrition Examination Survey 2011-2012. J Clin Lipidol. 2016;10(5):1109-18.

8. Yang Q, Zhong Y, Gillespie C, et al. Assessing potential population impact of statin treatment for primary prevention of atherosclerotic cardiovascular diseases in the USA: population-based modeling study. BMJ. 2017;7(1):e011684.

9. Dorsch MP, Lester CA, Ding Y, Joseph M, Brook RD. Effects of race on statin prescribing for primary prevention with high atherosclerotic cardiovascular disease risk in a large healthcare system. J Am Heart Assoc. 2019;8(22):e014709.

10. Gu A, Kamat S, Argulian E. Trends and disparities in statin use and low-density lipoprotein cholesterol levels among U.S. patients with diabetes, 1999-2014. Diabetes Res Clin Pract. 2018;139:1-10.

11. Ofori-Asenso R, Jakhu A, Zomer E, et al. Adherence and persistence among statin users aged 65 years and over: a systematic review and meta-analysis. J Gerontol A Biol Sci Med Sci. 2018;73(6):813-19.

12. Centers for Disease Control and Prevention. United States Diabetes Surveillance System. 2020. Accessed February 11, 2021. https://gis.cdc.gov/ grasp/diabetes/diabetesatlas.html
13. Davis AM, Taitel MS, Jiang J, et al. A national assessment of medication adherence to statins by the racial composition of neighborhoods. J Racial Ethn Health Disparities. 2017;4(3):462-71.

14. Colantonio LD, Rosenson RS, Deng L, et al. Adherence to statin therapy among U.S. adults between 2007 and 2014. J Am Heart Assoc. 2019;8(1):e010376.

15. Rodriguez F, Maron DJ, Knowles JW, Virani SS, Lin S, Heidenreich PA. Association of statin adherence with mortality in patients with atherosclerotic cardiovascular disease. JAMA Cardiol. 2019;4(3):206-13.

16. Brennan MB, Huang ES, Lobo JM, et al. Longitudinal trends and predictors of statin use among patients with diabetes. J Diabetes Complications. 2018;32(1):27-33.

17. Salami JA, Warraich H, Valero-Elizondo J, et al. National trends in statin use and expenditures in the U.S. adult population from 2002 to 2013: insights from the Medical Expenditure Panel Survey. JAMA Cardiol. 2017;2(1):56-65.

18. Pérez C, Ailshire JA. Aging in Puerto Rico: a comparison of health status among island Puerto Rican and mainland U.S. older adults. J Aging Health. 2017;29(6):1056-78.

19. Salas A, Acosta D, Ferri CP, et al. The prevalence, correlates, detection and control of diabetes among older people in low and middle income countries. A 10/66 Dementia Research Group Population-Based Survey. PloS One. 2016;11(2):e0149616.

20. Pabon-Nau LP, Cohen A, Meigs JB, Grant RW. Hypertension and diabetes prevalence among U.S. Hispanics by country of origin: the National Health Interview Survey 2000-2005. J Gen Intern Med. 2010;25(8):847-52.

21. Qato DM, Lee TA, Durazo-Arvizu R, et al. Statin and aspirin use among Hispanic and Latino adults at high cardiovascular risk: findings from the Hispanic Community Health Study/ Study of Latinos. J Am Heart Assoc. 2016;5(4):e002905. 
22. Qureshi WT, Kaplan RC, Swett K, et al. American College of Cardiology/ American Heart Association (ACC/AHA) Class I Guidelines for the Treatment of Cholesterol to Reduce Atherosclerotic Cardiovascular Risk: implications for U.S. Hispanics/Latinos based on findings from the Hispanic Community Health Study/ Study of Latinos (HCHS/SOL). J Am Heart Assoc. 2017;6(5):e005045.

23. Cheng YJ, Kanaya AM, Araneta MRG, et al. Prevalence of diabetes by race and ethnicity in the United States, 2011-2016. JAMA. 2019;322(24):2389-98.

24. Sattler EL, Lee JS, Perri M 3rd. Medication (re)fill adherence measures derived from pharmacy claims data in older Americans: a review of the literature. Drugs Aging. 2013;30(6):383-99.
25. Menke A, Casagrande S, Geiss L, Cowie CC. Prevalence of and trends in diabetes among Adults in the United States, 1988-2012. JAMA. 2015;314(10):1021-29.

26. Lin I, Sung J, Sanchez RJ, et al. Patterns of statin use in a real-world population of patients at high cardiovascular risk. J Manag Care Spec Pharm. 2016;22(6):685-98. doi: 10.18553/ jmcp.2016.22.6.685

27. Marcum ZA, Driessen J, Thorpe CT, Gellad WF, Donohue JM. Effect of multiple pharmacy use on medication adherence and drug-drug interactions in older adults with Medicare Part D. J Am Geriatr Soc. 2014;62(2):244-52.

28. Xie Z, St Clair P, Goldman DP, Joyce G. Racial and ethnic disparities in medication adherence among privately insured patients in the United States. PloS One. 2019;14(2):e0212117.
29. Clough JD, Martin SS, Navar AM, et al. Association of primary care providers' beliefs of statins for primary prevention and statin prescription. J Am Heart Assoc. 2019;8(3):e010241-e010241.

30. Cheetham TC, Niu F, Green K, et al. Primary nonadherence to statin medications in a managed care organization. J Manag Care Pharm. 2013;19(5):367-73. doi: 10.18553/jmcp.2013.19.5.367

31. Hernández-Muñoz JJ, Wei W, Sierra-Zorita R. Prevalence of rheumatoid arthritis and drug dispensing patterns among medicaid and Medicaid-Medicare dually eligible in Puerto Rico. Arthritis Care Res. 2021;73(2):199-206 . 